\title{
Evaluation of combined treatments of natural fibers: kenaf, abaca and oil palm fibers using micromechanical and SEM methods
}

\begin{abstract}
This paper presents the results of three different fibers, Kenaf (hibiscus cannabinus), oil palm (Elaeis guineensis) and abaca (Musa textilis) which were treated using 5 different types of surface treatments namely sodium hydroxide for 24 hours, glycidoxypropyltrimethoxy-silane for 24 hours, $\mathrm{NaOH}$ followed by Silane (each 24 hours), $\mathrm{NaOH}$ followed by Silane (each 12 hours) and $\mathrm{NaOH}$ followed by Silane (each 6 hours). The mechanical strength of the fibers were then studied and compared to the untreated fibers. The highest strength was given by the fibers treated with $\mathrm{NaOH}$ followed by the combined treatments and the lowest were the fibers treated with silane. Also the bonding strength between the fibers and unsaturated polyester resin were then evaluated using micro droplet pull out tests. It was proven that all the treatments improved on the bonding strength. The highest was silane followed by $\mathrm{NaOH}$. For the combined treatments it was found that the highest IFSS was given by the lowest treatment duration at 6 hours, due to the alkali nature of $\mathrm{NaOH}$ corroding the fibers which were then filled with silane, thus losing the ability to properly bond with the resin. The samples were also then characterized using a SEM to check the surface morphology which revealed that the $\mathrm{NaOH}$ reduced the diameter by removing impurities and lignin, thus increasing the aspect ratio. While silane coats the surface, increases the diameter and reduces the aspect ratio.
\end{abstract}

Keyword: Abaca; Bonding strength; Chemical treatment; Droplet test; Kenaf; OPF; SEM 